\title{
Das Siebente Gebot, das Hohelied und Calvin ${ }^{1}$
}

\author{
James Alfred Loader ${ }^{2}$ \\ Institut für Alttestamentliche Wissenschaft und Biblische Archäologie \\ Universität Wien \\ Professor Extraordinarius, UNISA
}

\begin{abstract}
The seventh commandment, Calvin and Canticles

Taking the Seventh Commandment (according to the numbering of the Calvinist tradition) as its focus, this article examines the hermeneutical principles of the Reformer, John Calvin, for the exposition of the Decalogue. This is related to a consideration of the 'theological meaning' of the Book of Canticles (Song of Songs), an Old Testament book which, significantly, does not feature among the commentaries of Calvin. It is argued that the secular impact of the erotic songs is equally strong and meaningful against the backdrop of Hellenistic Jewry, as it is against that of preexilic Israel. It is concluded that the dimension of the Song's sexual ethic is not an abrogation of the Law, but an extension of it: Here it is not a matter of mere prohibition and consequently, artificial techniques are not necessary in order to distil positive meaning from the negative commandment. Neither is this sexual ethic a matter of exclusive male rights, nor is purity protected at the cost of justice - Eros is taken up in Agape.
\end{abstract}

In seinem jüngsten $\mathrm{Buch}^{3}$, das als eine Art Nachtrag zu seinem unmittelbar zuvor erschienenen opus magnum ${ }^{4}$ zum theologischen Umgang mit dem Alten Testament wirkt,

\footnotetext{
1 Überarbeitete Fassung eines Impulsreferats im Rahmen des Symposions "Der Dekalog” an der Comenius-Universität Bratislava, 8. Feber 2001.

2 Der Autor lehrt an der Universität Wien als Ordinarius für Alttestamentliche Wissenschaft und Biblische Archäologie, ist auch Professor Extraordinarius im Department of Old Testament an der Universität von Südafrika, Pretoria.

3 History and Ideology in the Old Testament. Biblical Studies at the End of a Millennium, Oxford 2000, 179-180.

4 The Concept of Biblical Theology. An Old Testament Perspective, Minneapolis 1999.
} 
bringt James Barr den ganzen Skopus seiner Ausführungen zum folgenden Schluß: "All these questions come together as aspects of the apologetic task, which ... is now not so much the task of convincing unbelievers of what we believe, but rather the task of facing for ourselves as believers the uncertainties which are posed by recent discussion."

Das trifft in einem besonderen Sinne zu für die hier gebotenen Überlegungen zum Siebenten Gebot im Licht der Tatsache, daß es im gleichen Alten Testament wie das Hohelied steht. Zwar ist Hans-Peter Müller ${ }^{5}$ durchaus zuzustimmen, als er das Hohelied ein profan zu deutendes Buch nennt, "dem nicht aus anderen Teilen des Alten Testaments eine kanongerechte Theologie unterlegt werden darf', aber das bedeutet nicht, daß das theologische Aufarbeiten der Fragen und Unsicherheiten, die es im Rahmen der alttestamentlichen Diskussion aufruft, nicht auch "andere Teile des Alten Testaments" betrifft. Im vorliegenden Fall ist das 7. Gebot des Dekalogs (Ex. 20, 14; Dt. 5, 18) Gegenstand unseres Interesses, weil es in der Tradition als das Gebot, das die Sexualität regelt, verwendet worden ist und sogar das Grundgebot des Eros geworden ist. Aktuell ist die Frage nicht nur im Bereich der alttestamentlichen Wissenschaft, sondern auch interdisziplinär. Das wird sich sofort zeigen, indem wir mit der Auslegung von Johannes Calvin beginnen.

\section{DAS SIEBENTE GEBOT ALS GEBOT DER KEUSCHHEIT NACH CALVIN}

\subsection{Calvins Auslegungsprinzip für den Dekalog}

Nachdem er in seinem großen Kapitel zum Gesetz über die Heiligkeit und Integrität des mosaischen Gesetzes gesprochen hat, fährt Calvin folgendermaßen fort ${ }^{6}$ :

... es ist immer mehr in den Anordnungen und Verboten des Gesetzes gegeben als was in den Worten ausgedruckt ist. Dies ist aber so zu verstehen, daß es

\footnotetext{
5 Das Hohelied (ATD 16/2), Göttingen 1992, 8.

6 Institutio Religionis Christianae II.8.8 (Corpus Reformatorum XXX [Ioannis Calvini Opera II, Brunsvigae 1864] - Text der Ausgabe 1559).
} 
uns nicht wie lesbische Regeln wird ${ }^{7}$, so daß man der Schrift, indem man freizügig mit ihr umgeht, jedwede beliebige Bedeutung zuschreibt .... Wir sollen folgendermaßen von einem Grundsatz auf sein Gegenteil schließen: Wenn etwas Gott gefällig ist, ist sein Gegenteil ihm untauglich; wenn etwas ihm untauglich ist, ist sein Gegenteil ihm gefällig; wenn Gott etwas befiehlt, verbietet er das Gegenteil; wenn er etwas verbietet, befiehlt er das Gegenteil.

Etwas weiter ${ }^{8}$ meint Calvin, dieses Prinzip der "elliptischen Ausdrücke, die Umfangreicheres als das tatsächlich Gesagte bedeuten", folgendermaßen motivieren zu können:

Weil das Fleisch immer versucht, die Abscheulichkeit der Sünde zu verharmlosen ... führt der Herr das widerlichste und ungeheuerlichste in jeder Art von Verbrechen als Beispiele vor, so daß diese Vorgangsweise Zittern im Hörer hervorruft und einen größeren Ekel vor Sünde in seinen Geist abdrückt.

Obwohl damit das Argumentationsprinzip a majori ad minorem deutlich als hermeneutischer Grundsatz gegeben ist, scheinen mir auch weitere Grundsätze mit einbegriffen zu sein. Nicht nur wird die konsequenteste Form einer Sünde angegeben, damit alle minderen Formen einer Verbrechensart (transgressionis genus) implizit mitgegeben werden, sondern zumindest zwei weitere Kategorien spielen eine gravierende Rolle: Einmal wird Gegensätzlichkeit als Auslegungsschlüssel verwendet, und zum anderen wird das mit einem Kategoriewechsel von negativ und positiv verbunden. Das heißt aber, daß die eben zitierte Begründung für die apodiktische Form der Zehn Gebote nicht ausreicht - nur das erste Prinzip kann logisch damit abgedeckt werden (also, daß Gott hart formuliert, um alle Sünden in Zehn Geboten abdecken zu können). Damit öffnet sich Calvin die Möglichkeit, trotz seiner oben zitierten Abkehr von willkürlicher Schriftauslegung und von der Allegorese als "delirium" dem Text einen sensum

\footnotetext{
7 "Ne sit nobis instar lesbiae regulae." Diese Phrase fehlt im französischen Text (vgl. H. Beveridge, The Institutes of the Christian Religion by John Calvin Vol. 1, Grand Rapids 1964, 322, der allerdings ohne instar zitiert).

8 Inst. II.8.10.
} 
pleniorem zuschreiben zu können. Das ist schon deshalb pikant, weil ihm diese Vorgangsweise ermöglicht, gerade die "leibliche" oder "natürliche" Bedeutung des 7. Gebotes viel weiter als das von der semantischen Ebene des hebräischen Textes Zugelassene zu verfolgen - obwohl er nicht einen Kommentar zum Hohenlied geschrieben hat, wo ihn eine ähnliche Suche nach dem sensus plenior gerade von der "leiblichen" Bedeutung weggeführt hätte. Calvin verwendet also eine Methode, die er grundsätzlich ablehnt, um die biblische Grundlage für eine Sexualethik aus dem Dekalog gewinnen zu können, eine Methode aber, die es völlig unmöglich macht, diese Ethik auf das biblische Buch der Bücher über den Eros, das Hohelied, zu fundieren. Diesem Gefüge wenden wir uns nun zu.

Neben diesen hermeneutischen Grundsätzen wendet Calvin immer wieder das Prinzip an, daß der eigenen Willkür nicht freie Zügel gelassen werden sollen, indem ein Bibeltext im Licht der ganzen Bibel (gemeint ist: im Licht von anderen Texten der Bibel) ausgelegt werden soll ${ }^{9}$. Bekanntlich wurde dieses Prinzip in der nach-calvinischen Orthodoxie zu einem tragenden Pfeiler der biblischen Hermeneutik ausgebaut. Es wird sich aber unten herausstellen, daß die Subjektivität nicht so einfach zu eliminieren ist und daß Calvins Auslegung des 7. Gebotes gerade dadurch in Schwierigkeiten kommt.

\subsection{Die Anwendung in der Auslegung des 7. Gebotes}

Wenn es in Ex. 20,14 heißt, לא תנאי so bedeutet das einfach: "Du (männlich) sollst nicht unzulässigen Geschlechtsverkehr haben". Nur der Mann ist angesprochen. Das heißt, ein Mann darf nicht mit der Ehefrau eines anderen Mannes Geschlechtsverkehr haben, weil so die ehelichen Rechte einer dritten (männlichen) Partei verletzt werden ${ }^{10}$. Dazu gehört nach dem deuteronomistischen Gesetz auch das Recht eines verlobten

9 Im Fall des Dekalogs formuliert in Inst. II.8.8, aber freilich überall in seinen Werken von Calvin gebraucht.

10 Lev. 18, 20; 20, 10; Dt. 22, 22; in Spr. 6, 34 ist von der Rache des beleidigten Ehemannes die Rede. Vgl. D.N. Freedman \& B.E. Willoughby, Art. נאי, ThWAT V (1986), 123-129 (hier 124-125). Gleiches gilt dem Verb moechor, das in der Vulgata verwendet wird (non moechaberis). Karl Barth, Kirchliche Dogmatik III/4, Zürich 1957, 260-261, hält diese Auslegung des 7. Gebotes für richtig, wenn es "abstrakt" gemacht wird, was für ihn heißt, daß es "außerhalb" seines Zusammenhanges mit dem Bunde verstanden" wird. 
Mannes, weil Verkehr eines anderen mit seiner Verlobten die "Reinheit", auf die er bei einer Jungfrau ein Recht hat, antasten würde und daher sowohl in der deuteronomistischen als auch in der priesterlichen Gesetzgebung bestraft wird ${ }^{11}$. Dem Mann werden weder Besuche bei Prostituierten noch außerehelicher Verkehr verboten, und er wird nur im Fall der Rechtsverletzung ebenso wie die betroffene freiwillige Frau bestraft $^{12}$. Diese Rechtslage wird mehrfach in den Erzählungen der Genesis vorausgesetzt $^{13}$.

Das 7. Gebot hat also mit außerehelichem Geschlechtsverkehr, Keuschheit oder gar mit Sexualität im Allgemeinen nichts zu tun, sondern fordert Respekt für die Ehe als Gewährleistung des männlichen Rechts auf die Reinheit einer Ehefrau. Das Gebot verbietet Ehebruch im möglichst buchstäblichen Sinne, wo die Ehe eines Mannes durch einen anderen gebrochen bzw. beschädigt wird.

Das sieht Calvin, indem er am Anfang seiner Auslegung des Gebotes ${ }^{14}$ sagt, daß der Gegenstand, der spezifisch verboten wird, scortatio, Ehebruch in dem eben beschriebenen Sinne ist. Aber sein primäres hermeneutisches Prinzip für die Auslegung des Dekalogs ermöglicht ihm die Ausweitung der Bedeutung. Davon ausgehend, daß Ehebruch im technischen Sinn die schlimmste Form der Selbstbeschmutzung ist (,weil er sogar den Körper befleckt”), leitet er a majori ad minorem ab, „daß wir so disponiert werden, jede andere Form von Wollust zu ächten”. Daher seien alle andere Formen des

11 Dt. 22, 23f.; Lev. 18,30; zum Thema im Allgemeinen, vgl. E. Otto, Theologische Ethik des Alten Testaments, Stuttgart 1994, 39-47 (Literatur!); zur Frage nach der Art der Strafe und ihre Durchsetzbarkeit, vgl. H. McKeating, Sanctions against Adultery in Ancient Israelite Society, with some reflections on Methodology in the Study of Old Testament Ethics, JSOT 11 (1979), 57-72, die Reaktion von A. Phillips, Another look at Adultery, JSOT 20 (1981), 3-25, sowie die Antwort von McKeating, ebd., 25-26. Vgl. weiters die Betonung des Reinheitsprinzips im Heiligkeitsgesetz, Lev. 18,20.23f.25.27f.30. Walter Brueggemann, Theology of the Old Testament. Testimony, Dispute, Advocacy, Minneapolis 1997, 195, dürfte - gerade in einem Zusammenhang, wo von sexuellem Verhalten die Rede ist - Recht haben, daß Reinheit ("purity issues") grundlegender als Gerechtigkeit ("justice issues") ist, hier aber sind die beiden Kategorien aufs engste verbunden und zwar so, daß diese von jener abhängig ist; vgl. Erik H. Erikson, Identity and the Life Cycle, New York 1980, 67-87.

12 Dt. 22, 22ff.; Ez. 16, 35ff.; darauf bezieht sich die Anklage der Schriftgelehrten Joh. 8, 5 (der schuldige männliche Partner wird aber nicht angeklagt, obwohl er nach V. 4 "auf frischer Tat" ertappt worden ist!). Die Weisheitsliteratur enthält nur Warnungen gegen die Torheit des Ehebruchs (Spr. 2, 16-19; 5, 3-14; 6, 23-7, 27), keine gesetzliche Bestrafung.

13

Z.B. Gen. 12, 10-20; 20, 1-18; 26, 1-11; auch in 38, 14f.24.26 geht es um das Recht des künftigen Leviratsehemannes, Schela.

14 Inst. II.8.41. 
für unkeusch gehaltenen Benehmens im 7. Gebot untersagt. Diese Sicht geht so weit, daß auch sexuelle Zurückhaltung in der Ehe und sogar Kleidung und Gesten entsprechend der Intention des Gesetzgebers in diesem Gebot geregelt seien ${ }^{15}$.

Aber auch die Prinzipien der Gegenseitigkeit von positiv und negativ und der Gegensätzlichkeit spielen am Anfang der Auslegung eine wichtige Rolle. Auch wenn das Gebot negativ formuliert (und daher eigentlich ein Verbot) ist, setzt Calvin voraus, daß seine Intention positiv ist, nämlich daß Gott Keuschheit (castitas, pudicitia) und Reinheit (puritas) liebt. Daraus schließt er, daß wir uns vor allen Formen von deren Gegenteilen hüten sollen. Das heißt aber auch, daß wir uns nicht nur vor Ehebruch hüten, sondern auch eine ganze Reihe von sexuellen Tugenden pflegen sollen. In diesem Rahmen kommt ein bedenklicher Zug des Calvinischen Eheverständnisses hervor. Auch wenn die Ehe noch so hoch gewürdigt wird ${ }^{16}$, ist sie im Grunde genommen eine Notinstitution und deshalb etwas Negatives. Sie ist nämlich eine notwendige remedium ${ }^{17}$, ein Medikament gegen die intemperantia, libido oder cupiditas $^{18}$, die sexuelle Lust. Die Ehe wird also herab gewertet als Zuchtmittel, um das "Brennen" der Lust, das mit dem Sündenfall entstand, einigermaßen in Grenzen zu halten. Es ist m.E. klar, daß Calvin eine äußerst leibfeindliche Auslegung des 7. Gebotes bietet.

Das Prinzip der Gegensätzlichkeit ist in einer Auslegung des 7. Gebotes auch nicht anwendbar, wie Calvin es sich vorstellt. Was ist das Gegenteil davon, Ehebruch zu begehen? Was soll also hier geboten sein? Die Ehe zu pflegen, also zu heiraten? - Das kann nicht sein, weil diejenige, die die Gabe der "Enthaltung" haben, nach Calvin nicht zur Ehe verpflichtet sind ${ }^{19}$. Wenn die jeweiligen Gegenteile der einzelnen Gebote nicht

15 Inst II.8.44 Anfang bzw. Mitte.

16 Nach Inst. II.8.41 wurde sie von Gott "gesegnet", aber im nächsten Satz wird gesagt, daß Gott sie eingestellt hat, um ungezügelter Wollust zuvor zu kommen. Das ist eine ontologische Aussage, mit der das Wesen der Ehe, unbeschadet der Frage, ob sie vor oder nach dem Sündenfall entstand, angedeutet wird.

17 Inst II.8.42 und 43; in 8.42 auch subsidium, "Zuflucht”, “Aushilfe" genannt.

18 Alle drei Termini werden in den vier Paragraphen der Auslegung des 7. Gebotes (8.41-44) verwendet.

19 Ebd. Vgl. z.B. auch das 6. Gebot: Was soll das Gegenteil von Töten sein? - Die Antwort, "Leben zu pflegen", wäre so vage, daß jeweils ein völlig verschiedenes Verständnis des Gebotes bzw. Anwendung des Prinzips möglich wäre, etwa Leben zu schützen oder Leben zu zeugen (dem negativum, Leben nicht zu beenden, entsprechen logisch die positiva, bestehendes Leben zu verlängern sowie nicht bestehendes Leben beginnen zu lassen). 
eindeutig zu bestimmen sind, ist der hermeneutische Schlüssel für ihre Auslegung nicht brauchbar.

Calvins größtes Problem in diesem Zusammenhang ist, daß sein grundlegendes Prinzip, die Schrift habe sich selbst auszulegen, seine Interpretation des 7. Gebotes unterminiert. Das sieht man, sobald diese mit dem biblischen Buch Hoheslied, dem Text par excéllence zum Thema, konfrontiert wird. Es ist kein Wunder, daß Calvin dem Hohenlied keinen eigenen Kommentar gewidmet hat. Er konnte nämlich aufgrund seiner Abneigung vor der Allegorese keinen sensus plenior in ihm suchen. Die Alternative wäre für ihn aber auch problematisch, weil er sich so mit der „natürlichen” Bedeutung erotischer Lieder, deren explizite Freude an der Sexualität seiner Auslegung des 7. Gebotes quer liegt, hätte beschäftigen müssen. Dem wenden wir uns nunmehr kurz zu.

\section{KEUSCHHEIT ALS NICHTTHEMA IM HOHENLIED}

$\mathrm{Daß}$ es im Hohenlied um die erotische Liebe zwischen Mann und Frau geht, wird in der neueren alttestamentlichen Wissenschaft mehrheitlich akzeptiert ${ }^{20}$. Zwar wurde die „natürliche” Auslegung noch bis spät in das 20. Jahrhundert bestritten - und diese Ansichten finden noch immer in der kirchlichen Verwendung des Buches ihre Vertreter aber die Frage ist nicht mehr, ob der erotische Aspekt im Buch eine Rolle spielt, sondern welche religiöse Relevanz dem zuzuschreiben ist.

a) Wenn es bei Otto Kaiser ${ }^{21}$ heißt, das Hohelied sei eine Kunstdichtung, in der die Liebe ,ästhetisierend remythisiert” wird, so muß das keineswegs bedeuten, daß den Liedern eine sakral-religiöse Dimension zugemutet wird. Das wird deutlich, wenn Kaiser sich grundsätzlich der Sicht Oswald Loretz' anschließt, die wir noch

20 Vgl. R.E. Murphy, The Song of Songs: Critical Biblical Scholarship vis-à-vis exegetical traditions, in J.T. Butler, E.W. Conrad \& B.C. Ollenburger (Hg.), Understanding the Word. Essays in Honor of Bernhard W. Anderson (JSOT.S 37), Sheffield 1985, 65.

21

O. Kaiser, Grundriß der Einleitung in die kanonischen und deuterokanonischen Schriften des Alten Testaments. Band 3: Die poetischen und weisheitlichen Werke, Gütersloh 1994, 35. Es geht Kaiser hier um das "konkrete Spiel der Liebenden" (vgl. seinen zustimmenden Verweis auf die unten noch zu erwähnenden Ausführungen Loretz' zum Sinn der "aürlichen” Auslegung: Kaiser, Einleitung in das Alte Testament. Eine Einführung in ihre Ergebnisse und Probleme. Gütersloh ${ }^{5}$ 1984, 365). 
zu beachten haben. Demgegenüber gehen die Gedichte des Buches nach H.-P. Müller $^{22}$ gerade um die "naturreligiöse Erfarung", wozu er Folgendes schreibt:

Die religiöse, wenn schon nicht kanonische Bedeutung des Hohenliedes sehen wir vielmehr darin, daß Reminiszenzen halbvergessenen Heidentums bei weitgehendem Geltungsverlust der bislang tragenden und verpflichtenden Offizialreligion, beim späten Verfall vor allem der klassischen Heilsgeschichtsentwürfe augenblickhaft am liebenden und geliebten Menschen aufscheinen, weil das Heilige einen neuen dichterischen Verwirklichungsraum in Elementarerfahrungen suchen $\mathrm{mu}^{23}$.

Ohne zu behaupten, daß dieser Weg dem Heiligen unzugänglich war, ist dem doch entgegen zu halten, daß das Heilige nicht bis zum Verfall der Offizalreligion warten mußte, sich so zu manifestieren. Einmal waren die "Reminiszenzen des Heidentums" immer eine Versuchung für Israel, der nicht nur das Volk, sondern sogar Könige oft unterlagen ${ }^{24}$. Umgekehrt wurden viele Gesetzesbestimmungen im Bereich des Sexuellen gerade nach dem Untergang gesammelt und im Sinne einer nach dem Exil als verfallen gedachten "Offizialreligion" redigiert ${ }^{25}$. Einerseits ist fraglich, ob die erotischen Aspekte des Heidentums wirklich in vorexilischen Zeiten halb vergessen waren. Andererseits ist zu überlegen, ob die klassischen Heilsgeschichtsentwürfe wirklich in den späten Exilszeiten verfielen oder nicht vielmehr gerade in dieser Epoche gepflegt und weiter entwickelt wurden. Es ist die Frage, ob eine theologische Verwendung eines "profan zu deutende[n] Buch[es]", die von "naturreligiösen Zwischentönen” ausgehen muß,

22 Das Hohelied (ATD 16/2), Göttingen 1992, 9. Vgl. auch seinen Aufsatz, Die lyrische Reproduktion des Mythischen im Hohenlied, ZThK 73 (1976), 23-41.

23 Das Hohelied, 8.

24 Vgl. Freedman \& Willoughby, ThWAT V (1986), 123-129, die v.a. Beispiele aus der Kritik der Propheten Jeremia und Ezechiel (127-128) und weitere Literatur zu verschiedenen Aspekten des Themas (123-124) vorführen; vgl. Jer. 3, 6-9; 5,7f.; 13, 27; 23, 9-14; Ez. 16, 35-43; 23 ,43-49. Zur Vorstellung vom reliösen Synkretismus mancher Könige als "Hurerei”, vgl. z.B. 2. Chr. 21,13 und 2. Kön. 9, 22.

25 Das 3. Jh. v.Chr. war ja auch nach Müller (a.a.O., 4), unbeschadet aller skeptischen Auflösung, eine Zeit der Gesetzesfrömmigkeit, an der das Judentum teilhatte. 
um doch noch eine "religiöse Bedeutung" zu erringen (so Müller), den Haupttenor, also die zentrale Botschaft der Sammlung eruieren kann. Wohl aber kann eine religiöse Interpretation verteidigt werden, die demgegenüber nicht von der Sexualität als Motiv ausgeht, sondern von der (den) Bedeutung(en), die dem Buch in den jeweiligen Gesellschaften, die es rezipiert und tradiert haben, gegeben $\operatorname{wurde}(\mathrm{n})^{26}$. Nicht nur die Intentionen der einzelnen Lieder, auch nicht die der Redaktion, sondern auch die, die weiter stromab in der gleichen Tradition erkennbar werden, sind zu respektieren. Zumindest die grundlegende Einsicht, die nach Murphy dahin tendiert, daß das Hohelied auch auf Gott und die Menschen verweist, ist anzunehmen. Aber diese liefern "surplus meaning", "more meaning than the literal historical sense given to it by the author(s)",27.

b) Jetzt gilt es zu fragen, ob eine religiöse Bedeutung des Buches zu eruieren wäre, die der der Autoren (einschließlich der Redaktoren) entspricht, nicht aber nur von Zwischentönen, sondern vom Haupttenor der Lieder ausgeht. Ich meine schon wenn wir ihre Säkularität ${ }^{28}$ unbedingt ernst nehmen. Ein unverkennbarer Aspekt des Haupttenors ist, daß von der erotischen Liebe durchaus weltlich gesungen wird. Wenn überhaupt von religiösen Tönen im Buch die Rede sein kann, dann nur nebenbei als von "Zwischentönen”, die somit für die Botschaft des Buches von weniger Tragkraft sein können. Aber die alles durchwaltende Säkularität der Lieder ist so auffallend, daß sie in jeder Bedeutung, die dem Buch zugesprochen wird, eine entsprechend zentrale Rolle spielen muß. Daher ist das, was das Buch nicht sagt, gravierend, nicht um ihm "eine kanongerechte Theologie" zu unterlegen (Müller), sondern um die Potenz von seinem Inhalt in den (zu unserem

\footnotetext{
${ }^{26}$ Vgl. dazu R.E. Murphy, a.a.O., 67, dem die Feststellung in diesem Zusammenhang wichtig ist, daß nicht nur die hermeneutische Theorie hierzu beiträgt, sondern auch die von der historisch-kritischen Wissenschaft herausgearbeiteten Ergebnisse darauf hindeuten, daß die Symbolik der Sexualität in der Bibel eine wichtige Rolle spielt, z.B. Hos. 1-3, Jes. 62,5 (vgl. auch Jes. 54,5; Jer. 3,1ff.; 2. Kor. 11,2; Eph. 5, 22 33; und Apc. 19, 7; 21, 2.9; 22, 17).

27 Murphy, a.a.O.

28 Darauf hat bereits Curt Kuhl, Das Hohelied und seine Deutung, ThR (NF) 9 (1937), 137-167 hingewiesen, auch wenn man seine Formulierung ("ohne Gott und ohne jede Religion" [141]) zu derb finden mag.
} 
Zweck interessanten) frühen religiösen Gemeinschaften, die seine Wirkungskraft erfahren haben, auf die Spur zu kommen.

Einen wichtigen Anstoß hierzu hat Loretz bereits 1966 gegeben. Er formuliert allerdings sehr pauschal, wenn es heißt ${ }^{29}$ : "Wie hat sich Israel zu dieser Vergöttlichung des Sexuellen in seiner Umwelt verhalten? Hier gibt es nur eine, und zwar eine äußerst eindeutige Antwort: das Volk Gottes macht gegen diese Überschätzung des Geschlechtlichen von seinem Glauben an Jahwe her Front."

Obwohl, wie wir gesehen haben, diese Front immer wieder vom "Volk" im Norden und im Süden sowie von seinen Führern gebrochen wurde, so daß die "Antwort" nicht wirklich so eindeutig war, wird das Argument deswegen nur noch stärker, weil die Versuchung, sich im Kulturland den sexuell bestimmten religiösen Praktiken zuzuwenden, die nicht nur in Kanaan und Phönizien, sondern auch in Ägypten und Mesopotamien unterhalten wurden ${ }^{30}$, sich immer wieder als zu stark zeigte. Auch wenn nur die hellenistische Zeit als Entstehungsperiode in Frage käme (was mir eine überzogene Gewichtung der auf diese Zeit hinweisenden literarischen Elemente im Hohenlied und daher sehr unwahrscheinlich scheint), würde dieser Zusammenhang angesichts der sexuellen Freizügigkeit der griechischen Kultur des 3. Jahrhunderts für Juden genauso aktuell bleiben, war doch das jüdische "Bürgertum" in dieser Zeit der Versuchung einer "religiös-ästhetisch unterlaufene Verherrlichung einer naturorientierten Lebensfreude" als Kompensation für Gleichgültigkeit gegenüber der offiziellen Religion ausgesetzt ${ }^{31}$.

Man kann also nicht von einer "Theologie des Buches" reden und das Hohelied daher auch nicht als religiöses Loblied der ehelichen Keuschheit deuten. Eben das ist aber das Bedeutsame des Hohenliedes. Es soll eine ungemein große Leistung heißen, daß altorientalische Lieder über die erotische Liebe so völlig "weltlich" sprechen, vor allem in der vorexilischen Zeit, als die einzelnen Lieder entstanden. Für Israel war die klassische Sünde gegen Jahwe, daß die Sexualität entprofanisiert wurde. Im Kulturland

29 Die theologische Bedeutung des Hohenliedes, BZ (NF) 10 (1966), 29-43 (v.a. 39ff.; Zitat: S. 39).

30 Literaturverweise bei Loretz, a.a.O., Anm. 36.

31 Müller, a.a.O., 4; ders., Neige der althebräischen "Weisheit”, ZAW 90 (1978), 254ff. 
ging alles um die Fruchtbarkeit von Pflanzen, Tieren und Menschen, und die Religion war davon ein kräftiger Ausdruck. Daher das Hineintragen der Sexualität in die Götterwelt, wie die Baal-, Anat- und Aschera-Mythen plastisch illustrieren, und in den Gottesdienst, wie die weitverbreitete sakrale "Prostitution" in Kanaan zeigt. Also wurde alles dies für Israel nach seinem Einzug aus der monotonen Wüste, wo es seine monotheistische Religion gelernt hatte, eine enorme Versuchung. Von da aus gewinnt der Ausdruck, Gott "die Treue brechen" kräftigen Sinn; buchstäblich lautet er, "von [Gott/ihm/dir] davonhuren”,32. Gleiches gilt der Vorstellung vom religiösen Synkretismus mancher Könige als "Hurerei",33 und die großartige Entwicklung dieser Gedanken von den Propheten Hosea, Jeremia und Hesekiel sowie die ausgedehnten Gesetze über sexuelles Verhalten in Israel ${ }^{34}$. "Von Jahwe davonhuren" heißt im Literalsinn an der sakralen Prostitution des Baalsdienstes teilzunehmen; Freedman und Willouhgby ist zuzustimmen, "daß der Ehebruch gegen JHWH den Ehebruch gegenüber dem eigenen Ehepartner darstellte" ${ }^{35}$. Vor diesem Hintergrund, die Sexualität völlig profan oder "weltlich" zu besingen, heißt, sie zu "entsakralisieren". Theologisch ist damit gesagt, daß der Sexualität nichts Göttliches innewohnt ${ }^{36}$. Die Sexualität soll also nicht überbewertet werden, nicht ein Mittelpunkt werden - also kein Konkurrent für Jahwe sein.

Das extatische Element, das deutlich in den Liedern hervorkommt, betont aber ebenso unverkennbar, daß die Sexualität etwas Paradiesisches ist. Nicht nur wird sie unbefangen besungen, also gepriesen und empfohlen, sondern in der Metaphorik sogar mit dem Paradies in Verbindung gebracht. Das sieht man an der Metapher des Gartens und des Genusses der Früchte - die an die Paradieserzählung erinnern ${ }^{37}$. Die erotische

32 זְנ, z.B. Ps. 73, 27.

33 Z.B. 2. Chr. 21, 13, vgl. 2. Kön. 9, 22.

34 Z.B. Lev. 18.

35 ThWAT V, 127.

36 Loretz, a.a.O., 41.

37 Z.B. Hld. 1,6; 2,15; 4,12ff.; 6,2.11; 7,8.9.12-14; 8,11f.; vergleichbar wären auch Jes. 5,1; 27, 2-3; Jer.12

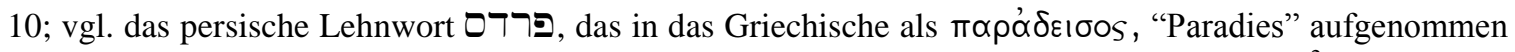
wurde. Zum Motiv der Rückkehr ins Paradies, vgl. O. Keel, Das Hohelied (ZBK 18), Zürich ${ }^{2}$ 1992, 233. 
Liebe soll also zwar keine Konkurrentin für Gott sein, ist aber da als Gabe, die man genießen soll. Damit wird auch einer im Orient diametral gegenüberstehenden Askese eine Grenze gesetzt. Die Sexualität wurde in der Zeit der Redaktion des Buches in der spätnachexilischen Zeit und danach auch unterschätzt, wie wir z.B. aus den Qumranschriften wissen. Damit steht das Buch auch "der leibes- und liebesfeindlichen Kultur diametral entgegen" 38 .

Immerhin liegt immanent im erotischen Lied auch die Warnung, daß die Liebe auch ihre eigene Gesetzmäßigkeit kennt. Die Liebe sei "stark wie der Tod" und gefährlich wie eine Gotteskraft $(8,6)$. Das heißt, daß die Liebe zwar nicht vergöttert werden soll, aber auch nicht irgendein Genuß ist ${ }^{39}$. Es haftet an ihr eine Macht, die respektiert werden soll. Dies kann freilich keineswegs als Keuschheit im Sinne des Verzichts auf außerehelichen Geschlechtsverkehr gedeutet werden. Von daher kann man vom Eros sprechen, der sogar über sich hinaus weist und demgemäß zur Verantwortung ruft. Auch so wird der Sexualität ihre Grenze gezeigt, was die Verantwortung des Menschen voraussetzt. Von daraus würde die "lichtlichkeit" des Hohenliedes ihre Aktualität auch in der sexuell freizügigen hellenistischen Zeit behalten. Die Kategorie der Verantwortung läßt sich gut mit der sozialen Dimension des Hohenliedes verbinden, auf die zunächst zu verweisen ist.

Neben der zweiseitigen Warnung vor Über- und Unterbewertung der Sexualität, liegt auch eine soziale Dimension in der Weise, in der die Lieder vom Eros sprechen oder - besser - singen. Das Buch sieht die Sexualität nicht nur als eine Funktion der Erzeugung von Nachkommenschaft. Damit ist ein antipatriarchalischer Zug angedeutet. So wirkt das Buch auch gegen eine soziale Organisation, die so ausgerichtet ist. Weiter

38 L. Schwienhorst-Schönberger, Das Hohelied, in: E. Zenger (Hg.), Einleitung in das Alte Testament, Stuttgart ${ }^{2} 1996,280$.

39 Sie soll nicht voreilig aufgeweckt werden (Hld. 8 ,4), sondern ihrer immanenten Gesetzmäßigkeit gehorchen. Vgl. H.-J. Heinevetter, “Komm nun, mein Liebster, Dein Garten ruft Dich!'Das Hohelied als programmatische Komposition (BBB 69) Frankfurt a.M. 1988, 190-198, auch wenn wir ihm nicht darin folgen müssen, daß er in Anschluß an F. Landy, Paradoxes of Paradise. Identity and Difference in the Song of Songs (BLS 7), 127, die Polarität von Tod und Leben als eigentliches Theologoumenon des Buches dahin interpretiert, daß die Liebe eine fast göttliche Macht sei, die sogar den Tod überwindet, was weder aus Hld. 8, 6 noch aus dem Buch als ganzem zu folgern ist (auch nicht mit der selbstaufhebenden Logik, daß die Liebe, um dem Tode in Kraft ebenbürtig zu sein, auch den Tod überwinden können muß, weil der Tod alles - auch die Liebe! - überwindet). 
gibt es nirgends im Alten oder im Neuen Testament ein Buch, in dem die Rollen von Mann und Frau so gleichberechtigt verteilt sind. Die Frau steht dem Mann als absolut gleichwertige Partnerin gegenüber. Damit wird nicht nur eine patriarchalische, sondern auch eine androzentrische Gesinnung des Einzelnen und der Gesellschaft zurückgewiesen $^{40}$. Angesichts der einseitigen Berücksichtigung der männlichen Rechte in den oben erwähnten gesetzlichen Regelungen Israels, läßt das Buch also auch eine Gegenstimme verlauten, die heute nicht weniger aktuell ist.

Eine symbolische oder "surplus"-Lesung (Murphy) oder sogar allegorische Lesung des Buches hat auch ihr Recht. Nicht weil das Buch gemeint war, etwas anders auszusagen als das, was da steht, sondern weil der Eros in der jüdischen und christlichen Tradition in der Tat auch eine Metapher für das Verhältnis von Gott und Mensch ist. Nicht nur Hos. 1, 2 und 3, oder Jer. 2 und 3 sowie Hsk. 16, sondern auch Eph. 5 und Apk. 21 verwenden diese Vorstellung. Wenn aber Christus der Bräutigam und die Gemeinde die Braut sind, dann nicht weil das Hohelied dafür ein Beleg ist, sondern weil man das Hohelied kreativ als zusätzliche Illustration der kirchlichen Glaubensmetaphorik verwendet ${ }^{41}$.

\section{FAZIT}

Während das 7. Gebot in apodiktischer Form das Verbrechen gegen das männliche Eherecht verbietet, spielt das Verbot im Hohenlied keine Rolle. An die Stelle des "Du sollst nicht" tritt das Zelebrieren, das "Laß uns ...!"42. Die Liebe wird gefeiert, und zwar die Liebe als Beseitigung der Restriktion und Diskriminierung, aber auch als Lebenskraft, die Verantwortung gegenüber Gott und gegenüber einander fordert. Also darf man

\footnotetext{
40 Ähnlich Keel, a.a.O., 232, wo er das Motiv der Rückkehr in das Paradies als Ausgleich des Verhältnisses zwischen Mann und Frau deutet: Das einseitige Begehren der Frau nach dem Mann, das von ihm dazu benützt werden kann, seine Herrschaft über sie auszuüben, wird damit überwunden, daß Mann und Frau nunmehr nach dem Hohenlied erotisch gleichermaßen aufeinander ausgerichtet sind und der fluchähnliche Zustand aufgehoben ist.

41 Vgl. auch O. Loretz, Zum Problem des Eros im Hohenlied, BZ (NF) 8 (1964), 191-216 (hier 215-216).

42 Vgl. das wiederholte Kohortativ in Hld. 7, $12 \mathrm{f}$.
} 
sagen, daß das, was im Hohenlied gefeiert wird, die den Eros umfassende Agape ist ${ }^{43}$. Daher enthält die sexualethische Dimension des Hohenliedes keine Annullierung des Gebotes, sondern seine Ausweitung: nicht mehr wird nur verboten, so daß man künstliche Techniken wie Gegensätzlichkeit braucht, um mit Calvin zu positiven Gesetzesnovellierungen $\mathrm{zu}$ kommen, nicht mehr wird ausschließlich das Recht des Mannes berücksichtigt, nicht mehr geht es auf Kosten der iustitia nur um puritas - es geht um eine Aufnahme des Eros in die Agape ${ }^{44}$. Wo nicht nur der Leib, sondern das Wesen von Mann und Frau füreinander das Herz der Liebe ist, ist nicht nur das Gesetz gegen Ehebruch, sondern das ganze Gesetz und die Propheten erfüllt.

Gesellschaftlich mag eine Polemik gegen Leibfeindlichkeit in der westlichen Welt schon obsolet sein (so Müller), aber das gilt weder flächendeckend für diese Gesellschaft noch für alle Gesellschaften der Welt und auch nicht für alle Formen des Phänomens "Leibfeindlichkeit". Es ist die Frage, ob die herrschende Sexualethik in der RömischKatholischen Amtskirche nicht der Calvins - horribile dictu - viel näher kommt als der des Hohenliedes und ob demgegenüber nicht doch etwas Polemisches, oder, wenn das zu aggressiv klingt, etwas Apologetisches im anfangs zitierten Barrschen Sinne zu sagen ist $^{45}$. Ist es nicht auch berechtigt $\mathrm{zu}$ fragen, ob wiederholte sexuelle Exzesse auf allen Ebenen einer zölibatären Gesellschaft, wo also die "Gabe der Enthaltung” nicht gegeben ist, anders als leibfeindlich von sowie gegenüber dem Täter aufzufassen sind. Es ist weiter $\mathrm{zu}$ fragen, ob Leibfeindlichkeit im geschlechtlichen Bereich überall in der muslimischen Welt obsolet ist und ob sie nicht in schrecklicher Form da ist, wo vor allem in Afrika die weibliche Beschneidung geübt wird. Schließlich ist es alles andere als

43 Auch in den Details sieht man das, z.B. in Hld. 1, 3: Der Name ist Ausprägung der Persönlichkeit; daher preist die Frau mit ihrem Salbe-Vergleich die Identität des Jünglings. Aber die Salbe ist ätherisch, sie wurde zu erotischen Zwecken von Frauen verwendet (Est. 2,12). Also sagt die Frau zunächst, daß der Jüngling gut riecht; im ersten Hemistichos wird also von wirklichem Öl gesprochen, das erotisch stimulierend empfunden wird. Dann folgt eine Erweiterung des Motivs, indem es im zweiten Hemistichos als Metapher verwendet wird; hier sagt die Frau, der Jüngling selbst (sein Name = sein Wesen) sei solches Ö1. Also nicht nur sein Duft, sondern sein Wesen selbst ist das Anregende. Damit ist ein Hinweis gegeben, daß die persönliche Bedeutung des Jünglings bzw. sein innerstes Wesen für sie bestimmend ist. Also: Liebe. Hier verbindet sich der Eros mit der Agape.

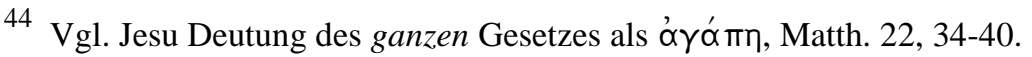

45 Statt Anschluß bei apologetischen Verfechtungen der, sagen wir einmal, "nicht-natürlichen" Auslegung des Hohenliedes (vgl. L. Krinetzki, Das Hohe Lied, Düsseldorf 1964, 31ff. und A. Feuillet, Einige 
obsolet, (selbst)kritisch Stellung zu nehmen zu einer verdinglichten Erotik, wo Freude am Banalen und an der rücksichtslosen Körperlichkeit vielleicht der leibfeindlichste Aspekt der westlichen Sexualauffassung ist. Zu alle dem ist Grundlegendes zu sagen. In der Tonart des Hohenliedes.

Scheinbare Widersprüche des Hohenliedes, BZ (NF) 8 (1964), 216-239, hier 238, wo einer "natürlichen" Auslegung "falsche apologetische Gründe" zugeschrieben wird). 